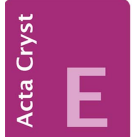
COMMUNICATIONS

ISSN 2056-9890

\section{Crystal structure of pencycuron}

\author{
Gihaeng Kang, Jineun Kim,* Eunjin Kwon and Tae Ho \\ Kim*
}

Department of Chemistry and Research Institute of Natural Sciences, Gyeongsang, National University, Jinju 660-701, Republic of Korea. *Correspondence e-mail:

thkim@gnu.ac.kr, jekim@gnu.ac.kr

Received 27 June 2015; accepted 29 June 2015

Edited by W. T. A. Harrison, University of Aberdeen, Scotland

In the title compound [systematic name: 1-(4-chlorobenzyl)-1cyclopentyl-3-phenylurea], $\mathrm{C}_{19} \mathrm{H}_{21} \mathrm{ClN}_{2} \mathrm{O}$, which is a urea fungicide, the cyclopentyl ring adopts an envelope conformation, with one of the methylene $\mathrm{C}$ atoms adjacent to the $\mathrm{C}$ atom bonding to the $\mathrm{N}$ atom as the flap. The dihedral angles between the mean planes of the central cyclopentyl ring (all atoms) and the chlorobenzyl and phenyl rings are 77.96 (6) and $57.77(7)^{\circ}$, respectively. In the crystal, $\mathrm{N}-\mathrm{H} \cdots \mathrm{O}$ hydrogen bonds link adjacent molecules, forming $C(4)$ chains propagating along the $b$-axis direction. The chains are linked by weak $\pi-\pi$ interactions between the chlorobenzene rings [centroid-centroid separation $=3.9942(9) \AA]$, resulting in two-dimensional networks extending parellel to the (110) plane.

Keywords: crystal structure; pencycuron; urea; fungicide; hydrogen bonding; $\pi-\pi$ interactions.

CCDC reference: 1409194

\section{Related literature}

For information on the fungicidal properties of the title compound, see: Pal et al. (2005). For a related crystal structure, see: Bjerglund et al. (2012).

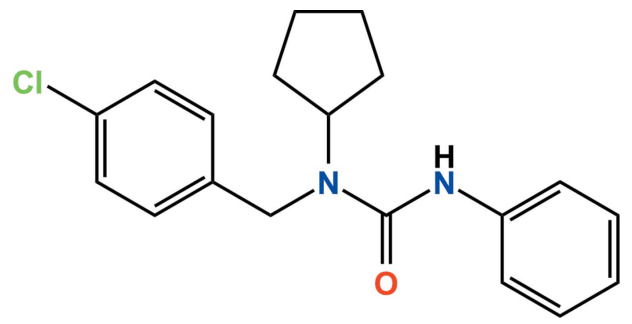

\section{Experimental}

2.1. Crystal data

$\mathrm{C}_{19} \mathrm{H}_{21} \mathrm{ClN}_{2} \mathrm{O}$

$M_{r}=328.83$

Orthorhombic, $\mathrm{Pbca}$

$a=12.1585(5) \AA$

$b=8.6721(4) \AA$

$c=32.6152(12) \AA$

$V=3438.9(2) \AA^{3}$

$Z=8$

Mo $K \alpha$ radiation

$\mu=0.23 \mathrm{~mm}^{-1}$

$T=173 \mathrm{~K}$

$0.50 \times 0.11 \times 0.09 \mathrm{~mm}$

\subsection{Data collection}

Bruker APEX-II CCD

diffractometer

Absorption correction: multi-scan

(SADABS; Bruker, 2009)

$T_{\min }=0.894, T_{\max }=0.980$

27037 measured reflections 3374 independent reflections 2698 reflections with $I>2 \sigma(I)$

$R_{\text {int }}=0.039$

\subsection{Refinement}

$R\left[F^{2}>2 \sigma\left(F^{2}\right)\right]=0.037$

$w R\left(F^{2}\right)=0.095$

$S=1.04$

3374 reflections

212 parameters

$\mathrm{H}$ atoms treated by a mixture of independent and constrained refinement

$\Delta \rho_{\max }=0.17{\mathrm{e} \AA^{-3}}^{-3}$

$\Delta \rho_{\min }=-0.24{\mathrm{e} \AA^{-3}}^{-3}$

Table 1

Hydrogen-bond geometry $\left(\AA,^{\circ}\right)$.

\begin{tabular}{lllll}
\hline$D-\mathrm{H} \cdots A$ & $D-\mathrm{H}$ & $\mathrm{H} \cdots A$ & $D \cdots A$ & $D-\mathrm{H} \cdots A$ \\
\hline $\mathrm{N} 2-\mathrm{H} 2 N \cdots \mathrm{O} 1^{\mathrm{i}}$ & $0.828(19)$ & $2.081(19)$ & $2.8838(17)$ & $163.1(17)$ \\
\hline
\end{tabular}

Symmetry code: (i) $-x+\frac{1}{2}, y+\frac{1}{2}, z$.

Data collection: APEX2 (Bruker, 2009); cell refinement: SAINT (Bruker, 2009); data reduction: $S A I N T$; $\operatorname{program}(\mathrm{s})$ used to solve structure: SHELXS97 (Sheldrick 2008); program(s) used to refine structure: SHELXL2013 (Sheldrick, 2015); molecular graphics: DIAMOND (Brandenburg, 2010); software used to prepare material for publication: SHELXTL (Sheldrick 2008).

\section{Acknowledgements}

This research was supported by the Basic Science Research Program through the National Research Foundation of Korea (NRF) funded by the Ministry of Education, Science and Technology (No. 2015R1D1A4A01020317).

Supporting information for this paper is available from the IUCr electronic archives (Reference: HB7456).

\section{References}

Bjerglund, K., Lindhardt, A. T. \& Skrydstrup, T. (2012). J. Org. Chem. 77, 3793-3799.

Brandenburg, K. (2010). DIAMOND. Crystal Impact GbR, Bonn, Germany.

Bruker (2009). APEX2, SAINT and SADABS. Bruker AXS Inc., Madison, Wisconsin, USA.

Pal, P., Chakrabarti, K., Chakraborty, A. \& Chowdhury, A. (2005). Pest. Manag. Sci. 61, 1220-1223.

Sheldrick, G. M. (2008). Acta Cryst. A64, 112-122.

Sheldrick, G. M. (2015). Acta Cryst. C71, 3-8. 


\section{supporting information}

Acta Cryst. (2015). E71, o532 [https://doi.org/10.1107/S2056989015012414]

\section{Crystal structure of pencycuron}

\section{Gihaeng Kang, Jineun Kim, Eunjin Kwon and Tae Ho Kim}

\section{S1. Comment}

Pencycuron, [systematic name: 1-(4-chlorobenzyl)-1-cyclopentyl-3-phenylurea], is a urea fungicide and it has been used for the control of diseases in various crops, including rice (Pal et al., 2005). However, until now its crystal structure has not been reported. In the title compound (Fig. 1), the dihedral angles between the mean planes of the central cyclopentyl ring [r.m.s. deviation $=0.1693$ ] and the chlorobenzyl and phenyl rings are $77.96(6)$ and $57.778(7)^{\circ}$, respectively. All bond lengths and bond angles are normal and comparable to those observed in the crystal structure of a similar compound (Bjerglund et al., 2012).

In the crystal (Fig. 2), $\mathrm{N}-\mathrm{H} \cdots \mathrm{O}$ hydrogen bonds link adjacent molecules, forming a one-dimensional chains along the $b$ axis direction (Table. 1). The chains are linked by weak $\pi-\pi$ interactions $\left[C g 1 \cdots C g 1^{1 i}, 3.9942\right.$ (9) $\left.\AA\right]$, resulting in a twodimensional networks parellel to the (110) plane ( $C g 1$ is the centroid of the $\mathrm{C} 1-\mathrm{C} 6$ ring)[for symmetry codes: (ii), $-x,-y,-$ $z]$.

\section{S2. Experimental}

The title compound was purchased from the Dr Ehrenstorfer GmbH Company. Slow evaporation of a solution in $\mathrm{CH}_{2} \mathrm{Cl}_{2}$ gave colourless needles.

\section{S3. Refinement}

The N-bound $\mathrm{H}$ atom was located in a difference Fourier map and freely refined $(\mathrm{N}-\mathrm{H}=0.828$ (19) $\AA$ ). The $\mathrm{C}$-bound $\mathrm{H}$ atoms were positioned geometrically and refined using a riding model with $\mathrm{d}(\mathrm{C}-\mathrm{H})=1.00 \AA, U_{\text {iso }}=1.2 U_{\text {eq }}(\mathrm{C})$ for Csp $p^{3}$ $-\mathrm{H}, \mathrm{d}(\mathrm{C}-\mathrm{H})=0.99 \AA, U_{\text {iso }}=1.2 U_{\text {eq }}(\mathrm{C})$ for $\mathrm{CH}_{2}$ groups, $\mathrm{d}(\mathrm{C}-\mathrm{H})=0.95 \AA, U_{\text {iso }}=1.2 U_{\text {eq }}(\mathrm{C})$ for aromatic $\mathrm{C}-\mathrm{H}$. 


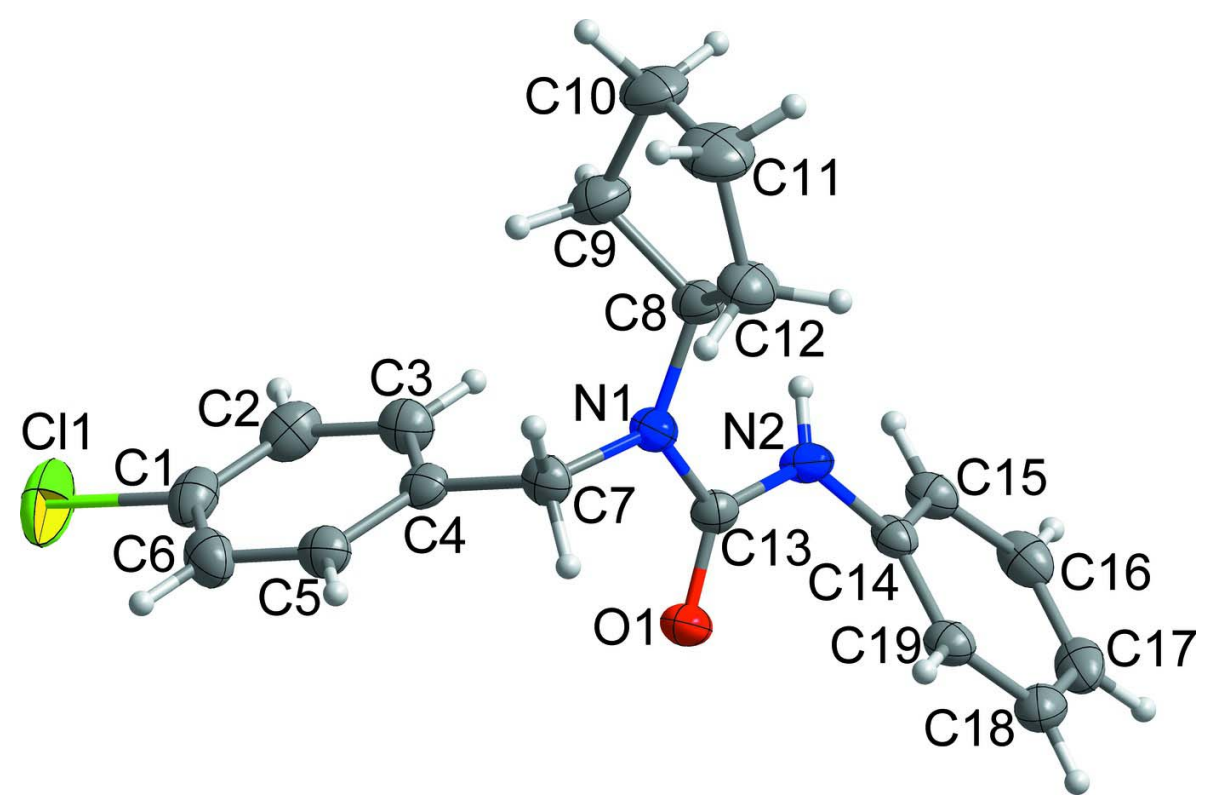

Figure 1

The asymmetric unit of the title compound with displacement ellipsoids drawn at the $50 \%$ probability level.

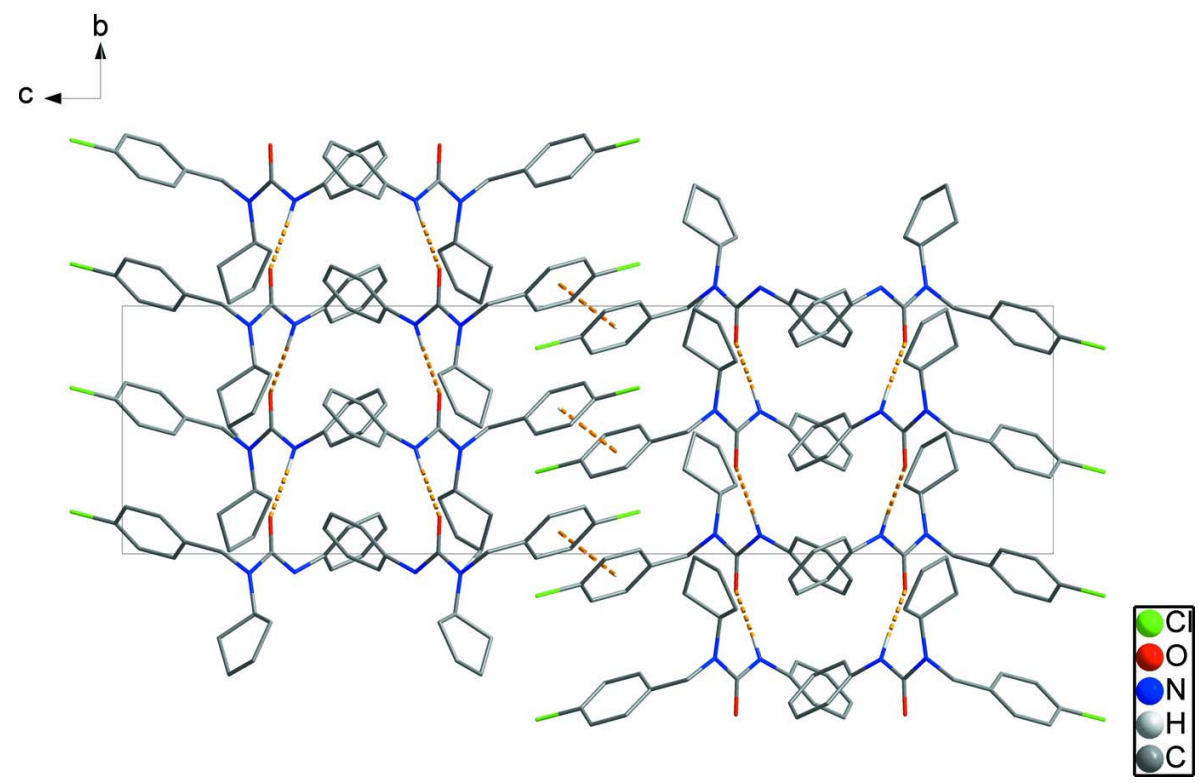

Figure 2

Crystal packing viewed along the $a$ axis. The intermolecular $\mathrm{N}-\mathrm{H} \cdots \mathrm{O}$ hydrogen bonds and weak $\pi-\pi$ interactions are shown as dashed lines.

1-(4-Chlorobenzyl)-1-cyclopentyl-3-phenylurea

Crystal data

$\mathrm{C}_{19} \mathrm{H}_{21} \mathrm{ClN}_{2} \mathrm{O}$

$M_{r}=328.83$

Orthorhombic, $\mathrm{Pbca}$

$a=12.1585$ (5) $\AA$

$$
\begin{aligned}
& b=8.6721(4) \AA \\
& c=32.6152(12) \AA \\
& V=3438.9(2) \AA^{3} \\
& Z=8
\end{aligned}
$$


$F(000)=1392$

$D_{\mathrm{x}}=1.270 \mathrm{Mg} \mathrm{m}^{-3}$

Mo $K \alpha$ radiation, $\lambda=0.71073 \AA$

Cell parameters from 6227 reflections

$\theta=2.5-27.3^{\circ}$

Data collection

Bruker APEX-II CCD diffractometer

$\varphi$ and $\omega$ scans

Absorption correction: multi-scan

(SADABS; Bruker, 2009)

$T_{\min }=0.894, T_{\max }=0.980$

27037 measured reflections

\section{Refinement}

Refinement on $F^{2}$

Least-squares matrix: full

$R\left[F^{2}>2 \sigma\left(F^{2}\right)\right]=0.037$

$w R\left(F^{2}\right)=0.095$

$S=1.04$

3374 reflections

212 parameters

0 restraints $\mu=0.23 \mathrm{~mm}^{-1}$

$T=173 \mathrm{~K}$

Needle, colourless

$0.50 \times 0.11 \times 0.09 \mathrm{~mm}$

3374 independent reflections

2698 reflections with $I>2 \sigma(I)$

$R_{\text {int }}=0.039$

$\theta_{\text {max }}=26.0^{\circ}, \theta_{\min }=2.1^{\circ}$

$h=-14 \rightarrow 14$

$k=-6 \rightarrow 10$

$l=-40 \rightarrow 40$

Hydrogen site location: mixed

$\mathrm{H}$ atoms treated by a mixture of independent and constrained refinement

$w=1 /\left[\sigma^{2}\left(F_{\mathrm{o}}{ }^{2}\right)+(0.0384 P)^{2}+1.2518 P\right]$

where $P=\left(F_{\mathrm{o}}^{2}+2 F_{\mathrm{c}}{ }^{2}\right) / 3$

$(\Delta / \sigma)_{\max }<0.001$

$\Delta \rho_{\max }=0.17 \mathrm{e} \AA^{-3}$

$\Delta \rho_{\min }=-0.24$ e $\AA^{-3}$

Special details

Geometry. All e.s.d.'s (except the e.s.d. in the dihedral angle between two 1.s. planes) are estimated using the full covariance matrix. The cell e.s.d.'s are taken into account individually in the estimation of e.s.d.'s in distances, angles and torsion angles; correlations between e.s.d.'s in cell parameters are only used when they are defined by crystal symmetry. An approximate (isotropic) treatment of cell e.s.d.'s is used for estimating e.s.d.'s involving 1.s. planes.

Fractional atomic coordinates and isotropic or equivalent isotropic displacement parameters $\left(\AA^{2}\right)$

\begin{tabular}{lllll}
\hline & $x$ & $y$ & $z$ & $U_{\text {iso }} * / U_{\text {eq }}$ \\
\hline C11 & $0.23590(4)$ & $-0.16966(8)$ & $-0.05434(2)$ & $0.06131(19)$ \\
O1 & $0.15878(8)$ & $-0.14846(11)$ & $0.15909(3)$ & $0.0287(2)$ \\
$\mathrm{N} 1$ & $0.08966(10)$ & $0.07872(15)$ & $0.13656(4)$ & $0.0278(3)$ \\
$\mathrm{N} 2$ & $0.23017(10)$ & $0.07453(16)$ & $0.18515(4)$ & $0.0288(3)$ \\
$\mathrm{H} 2 \mathrm{~N}$ & $0.2489(14)$ & $0.162(2)$ & $0.1777(5)$ & $0.039(5)^{*}$ \\
$\mathrm{C} 1$ & $0.17668(14)$ & $-0.1232(2)$ & $-0.00710(5)$ & $0.0384(4)$ \\
$\mathrm{C} 2$ & $0.22919(13)$ & $-0.0213(2)$ & $0.01841(5)$ & $0.0397(4)$ \\
$\mathrm{H} 2$ & 0.2975 & 0.0233 & 0.0106 & $0.048^{*}$ \\
$\mathrm{C} 3$ & $0.18127(12)$ & $0.0160(2)$ & $0.05576(5)$ & $0.0355(4)$ \\
$\mathrm{H} 3$ & 0.2173 & 0.0863 & 0.0736 & $0.043^{*}$ \\
$\mathrm{C} 4$ & $0.08150(12)$ & $-0.04807(17)$ & $0.06739(4)$ & $0.0277(3)$ \\
$\mathrm{C} 5$ & $0.03091(13)$ & $-0.15110(19)$ & $0.04097(5)$ & $0.0327(4)$ \\
$\mathrm{H} 5$ & -0.0373 & -0.1962 & 0.0486 & $0.039^{*}$ \\
$\mathrm{C} 6$ & $0.07769(14)$ & $-0.1895(2)$ & $0.00376(5)$ & $0.0390(4)$ \\
$\mathrm{H} 6$ & 0.0423 & -0.2604 & -0.0141 & $0.047^{*}$ \\
C7 & $0.02356(12)$ & $-0.00774(19)$ & $0.10708(4)$ & $0.0298(3)$ \\
H7A & -0.0428 & 0.0535 & 0.1005 & $0.036^{*}$ \\
H7B & -0.0012 & -0.1045 & 0.1203 & $0.036^{*}$
\end{tabular}




$\begin{array}{lllll}\text { C8 } & 0.06470(12) & 0.24352(18) & 0.14250(5) & 0.0315(4) \\ \text { H8 } & 0.1190 & 0.2854 & 0.1627 & 0.038^{*} \\ \text { C9 } & 0.07296(15) & 0.3403(2) & 0.10377(5) & 0.0436(4) \\ \text { H9A } & 0.0381 & 0.2873 & 0.0802 & 0.052^{*} \\ \text { H9B } & 0.1506 & 0.3629 & 0.0969 & 0.052^{*} \\ \text { C10 } & 0.01105(17) & 0.4869(2) & 0.11481(6) & 0.0532(5) \\ \text { H10A } & -0.0149 & 0.5406 & 0.0898 & 0.064^{*} \\ \text { H10B } & 0.0584 & 0.5579 & 0.1307 & 0.064^{*} \\ \text { C11 } & -0.08580(17) & 0.4315(2) & 0.14061(7) & 0.0565(5) \\ \text { H11A } & -0.1520 & 0.4182 & 0.1233 & 0.068^{*} \\ \text { H11B } & -0.1027 & 0.5070 & 0.1625 & 0.068^{*} \\ \text { C12 } & -0.05145(14) & 0.2774(2) & 0.15928(6) & 0.0421(4) \\ \text { H12A } & -0.1033 & 0.1949 & 0.1511 & 0.050^{*} \\ \text { H12B } & -0.0502 & 0.2841 & 0.1896 & 0.050^{*} \\ \text { C13 } & 0.15966(11) & -0.00577(17) & 0.16037(4) & 0.0249(3) \\ \text { C14 } & 0.29239(11) & 0.00718(17) & 0.21711(4) & 0.0255(3) \\ \text { C15 } & 0.39770(12) & 0.06323(18) & 0.22439(5) & 0.0300(3) \\ \text { H15 } & 0.4283 & 0.1387 & 0.2066 & 0.036^{*} \\ \text { C16 } & 0.45819(13) & 0.0096(2) & 0.25737(5) & 0.0357(4) \\ \text { H16 } & 0.5295 & 0.0500 & 0.2625 & 0.043^{*} \\ \text { C17 } & 0.41528(14) & -0.1025(2) & 0.28280(5) & 0.0381(4) \\ \text { H17 } & 0.4569 & -0.1396 & 0.3054 & 0.046^{*} \\ \text { C18 } & 0.31135(14) & -0.16039(19) & 0.27514(5) & 0.0354(4) \\ \text { H18 } & 0.2822 & -0.2390 & 0.2923 & 0.043^{*} \\ \text { C19 } & 0.24920(12) & -0.10478(18) & 0.24270(4) & 0.0293(3) \\ \text { H19 } & 0.1772 & -0.1434 & 0.2381 & 0.035^{*} \\ & & & & \end{array}$

Atomic displacement parameters $\left(\AA^{2}\right)$

\begin{tabular}{lllllll}
\hline & $U^{11}$ & $U^{22}$ & $U^{33}$ & $U^{12}$ & $U^{13}$ & $U^{23}$ \\
\hline C11 & $0.0543(3)$ & $0.0950(5)$ & $0.0346(2)$ & $0.0261(3)$ & $0.0028(2)$ & $-0.0022(3)$ \\
O1 & $0.0304(5)$ & $0.0214(5)$ & $0.0343(6)$ & $-0.0008(5)$ & $-0.0029(4)$ & $-0.0001(5)$ \\
N1 & $0.0293(6)$ & $0.0238(7)$ & $0.0303(6)$ & $0.0018(6)$ & $-0.0084(5)$ & $-0.0012(6)$ \\
N2 & $0.0295(7)$ & $0.0207(6)$ & $0.0361(7)$ & $-0.0033(6)$ & $-0.0097(5)$ & $0.0054(6)$ \\
C1 & $0.0388(9)$ & $0.0465(10)$ & $0.0298(8)$ & $0.0157(8)$ & $-0.0022(7)$ & $0.0049(8)$ \\
C2 & $0.0300(8)$ & $0.0476(11)$ & $0.0414(9)$ & $0.0029(8)$ & $0.0011(7)$ & $0.0082(9)$ \\
C3 & $0.0303(8)$ & $0.0365(9)$ & $0.0396(9)$ & $-0.0018(7)$ & $-0.0046(7)$ & $-0.0005(8)$ \\
C4 & $0.0274(7)$ & $0.0269(8)$ & $0.0290(7)$ & $0.0030(7)$ & $-0.0073(6)$ & $0.0041(7)$ \\
C5 & $0.0300(8)$ & $0.0331(9)$ & $0.0350(8)$ & $-0.0003(7)$ & $-0.0070(6)$ & $0.0014(7)$ \\
C6 & $0.0408(9)$ & $0.0412(10)$ & $0.0352(8)$ & $0.0044(8)$ & $-0.0113(7)$ & $-0.0056(8)$ \\
C7 & $0.0254(7)$ & $0.0316(9)$ & $0.0324(8)$ & $-0.0024(7)$ & $-0.0056(6)$ & $-0.0017(7)$ \\
C8 & $0.0354(8)$ & $0.0245(8)$ & $0.0345(8)$ & $0.0026(7)$ & $-0.0091(7)$ & $0.0022(7)$ \\
C9 & $0.0495(10)$ & $0.0358(10)$ & $0.0454(10)$ & $0.0022(8)$ & $-0.0033(8)$ & $0.0120(9)$ \\
C10 & $0.0705(13)$ & $0.0338(10)$ & $0.0552(11)$ & $0.0087(10)$ & $-0.0153(10)$ & $0.0118(9)$ \\
C11 & $0.0532(11)$ & $0.0433(12)$ & $0.0730(14)$ & $0.0190(10)$ & $-0.0099(10)$ & $0.0020(11)$ \\
C12 & $0.0459(10)$ & $0.0343(9)$ & $0.0459(10)$ & $0.0084(8)$ & $0.0025(8)$ & $-0.0006(8)$ \\
C13 & $0.0231(7)$ & $0.0246(8)$ & $0.0269(7)$ & $-0.0011(6)$ & $0.0017(6)$ & $0.0016(7)$ \\
C14 & $0.0265(7)$ & $0.0211(7)$ & $0.0290(7)$ & $0.0047(6)$ & $-0.0049(6)$ & $-0.0033(6)$
\end{tabular}




\begin{tabular}{lllllll}
$\mathrm{C} 15$ & $0.0278(8)$ & $0.0253(8)$ & $0.0369(8)$ & $0.0015(6)$ & $-0.0026(6)$ & $-0.0027(7)$ \\
$\mathrm{C} 16$ & $0.0271(7)$ & $0.0346(9)$ & $0.0454(9)$ & $0.0065(7)$ & $-0.0104(7)$ & $-0.0104(8)$ \\
$\mathrm{C} 17$ & $0.0430(9)$ & $0.0375(10)$ & $0.0339(8)$ & $0.0145(8)$ & $-0.0141(7)$ & $-0.0038(8)$ \\
$\mathrm{C} 18$ & $0.0440(9)$ & $0.0297(9)$ & $0.0326(8)$ & $0.0073(8)$ & $-0.0023(7)$ & $0.0018(7)$ \\
C19 & $0.0286(7)$ & $0.0249(8)$ & $0.0342(8)$ & $0.0023(7)$ & $-0.0038(6)$ & $-0.0013(7)$ \\
\hline
\end{tabular}

Geometric parameters $\left(\AA,{ }^{\circ}\right)$

\begin{tabular}{|c|c|c|c|}
\hline $\mathrm{C} 11-\mathrm{C} 1$ & $1.7478(17)$ & $\mathrm{C} 8-\mathrm{H} 8$ & 1.0000 \\
\hline $\mathrm{O} 1-\mathrm{C} 13$ & $1.2382(17)$ & $\mathrm{C} 9-\mathrm{C} 10$ & $1.521(3)$ \\
\hline $\mathrm{N} 1-\mathrm{C} 13$ & $1.3654(18)$ & C9-H9A & 0.9900 \\
\hline $\mathrm{N} 1-\mathrm{C} 7$ & $1.4604(18)$ & C9-H9B & 0.9900 \\
\hline $\mathrm{N} 1-\mathrm{C} 8$ & $1.474(2)$ & $\mathrm{C} 10-\mathrm{C} 11$ & $1.525(3)$ \\
\hline $\mathrm{N} 2-\mathrm{C} 13$ & $1.3686(19)$ & $\mathrm{C} 10-\mathrm{H} 10 \mathrm{~A}$ & 0.9900 \\
\hline $\mathrm{N} 2-\mathrm{C} 14$ & $1.4141(18)$ & $\mathrm{C} 10-\mathrm{H} 10 \mathrm{~B}$ & 0.9900 \\
\hline $\mathrm{N} 2-\mathrm{H} 2 \mathrm{~N}$ & $0.828(19)$ & $\mathrm{C} 11-\mathrm{C} 12$ & $1.527(3)$ \\
\hline $\mathrm{C} 1-\mathrm{C} 2$ & $1.371(2)$ & C11-H11A & 0.9900 \\
\hline $\mathrm{C} 1-\mathrm{C} 6$ & $1.380(2)$ & C11-H11B & 0.9900 \\
\hline $\mathrm{C} 2-\mathrm{C} 3$ & $1.389(2)$ & $\mathrm{C} 12-\mathrm{H} 12 \mathrm{~A}$ & 0.9900 \\
\hline $\mathrm{C} 2-\mathrm{H} 2$ & 0.9500 & $\mathrm{C} 12-\mathrm{H} 12 \mathrm{~B}$ & 0.9900 \\
\hline $\mathrm{C} 3-\mathrm{C} 4$ & $1.387(2)$ & $\mathrm{C} 14-\mathrm{C} 19$ & $1.384(2)$ \\
\hline $\mathrm{C} 3-\mathrm{H} 3$ & 0.9500 & $\mathrm{C} 14-\mathrm{C} 15$ & $1.390(2)$ \\
\hline $\mathrm{C} 4-\mathrm{C} 5$ & $1.385(2)$ & $\mathrm{C} 15-\mathrm{C} 16$ & $1.384(2)$ \\
\hline $\mathrm{C} 4-\mathrm{C} 7$ & $1.515(2)$ & $\mathrm{C} 15-\mathrm{H} 15$ & 0.9500 \\
\hline $\mathrm{C} 5-\mathrm{C} 6$ & $1.381(2)$ & $\mathrm{C} 16-\mathrm{C} 17$ & $1.380(2)$ \\
\hline $\mathrm{C} 5-\mathrm{H} 5$ & 0.9500 & $\mathrm{C} 16-\mathrm{H} 16$ & 0.9500 \\
\hline $\mathrm{C} 6-\mathrm{H} 6$ & 0.9500 & $\mathrm{C} 17-\mathrm{C} 18$ & $1.382(2)$ \\
\hline $\mathrm{C} 7-\mathrm{H} 7 \mathrm{~A}$ & 0.9900 & C17-H17 & 0.9500 \\
\hline C7-H7B & 0.9900 & $\mathrm{C} 18-\mathrm{C} 19$ & $1.387(2)$ \\
\hline $\mathrm{C} 8-\mathrm{C} 9$ & $1.520(2)$ & $\mathrm{C} 18-\mathrm{H} 18$ & 0.9500 \\
\hline $\mathrm{C} 8-\mathrm{C} 12$ & $1.543(2)$ & C19-H19 & 0.9500 \\
\hline $\mathrm{C} 13-\mathrm{N} 1-\mathrm{C} 7$ & $116.23(12)$ & $\mathrm{H} 9 \mathrm{~A}-\mathrm{C} 9-\mathrm{H} 9 \mathrm{~B}$ & 109.0 \\
\hline $\mathrm{C} 13-\mathrm{N} 1-\mathrm{C} 8$ & $125.02(12)$ & $\mathrm{C} 9-\mathrm{C} 10-\mathrm{C} 11$ & $104.42(15)$ \\
\hline $\mathrm{C} 7-\mathrm{N} 1-\mathrm{C} 8$ & $118.11(12)$ & $\mathrm{C} 9-\mathrm{C} 10-\mathrm{H} 10 \mathrm{~A}$ & 110.9 \\
\hline $\mathrm{C} 13-\mathrm{N} 2-\mathrm{C} 14$ & $124.07(13)$ & $\mathrm{C} 11-\mathrm{C} 10-\mathrm{H} 10 \mathrm{~A}$ & 110.9 \\
\hline $\mathrm{C} 13-\mathrm{N} 2-\mathrm{H} 2 \mathrm{~N}$ & $117.8(12)$ & $\mathrm{C} 9-\mathrm{C} 10-\mathrm{H} 10 \mathrm{~B}$ & 110.9 \\
\hline $\mathrm{C} 14-\mathrm{N} 2-\mathrm{H} 2 \mathrm{~N}$ & $116.5(12)$ & $\mathrm{C} 11-\mathrm{C} 10-\mathrm{H} 10 \mathrm{~B}$ & 110.9 \\
\hline $\mathrm{C} 2-\mathrm{C} 1-\mathrm{C} 6$ & $121.24(15)$ & $\mathrm{H} 10 \mathrm{~A}-\mathrm{C} 10-\mathrm{H} 10 \mathrm{~B}$ & 108.9 \\
\hline $\mathrm{C} 2-\mathrm{C} 1-\mathrm{C} 11$ & $119.44(13)$ & $\mathrm{C} 10-\mathrm{C} 11-\mathrm{C} 12$ & $106.55(15)$ \\
\hline $\mathrm{C} 6-\mathrm{C} 1-\mathrm{Cl1}$ & $119.32(14)$ & $\mathrm{C} 10-\mathrm{C} 11-\mathrm{H} 11 \mathrm{~A}$ & 110.4 \\
\hline $\mathrm{C} 1-\mathrm{C} 2-\mathrm{C} 3$ & $119.13(15)$ & $\mathrm{C} 12-\mathrm{C} 11-\mathrm{H} 11 \mathrm{~A}$ & 110.4 \\
\hline $\mathrm{C} 1-\mathrm{C} 2-\mathrm{H} 2$ & 120.4 & $\mathrm{C} 10-\mathrm{C} 11-\mathrm{H} 11 \mathrm{~B}$ & 110.4 \\
\hline $\mathrm{C} 3-\mathrm{C} 2-\mathrm{H} 2$ & 120.4 & $\mathrm{C} 12-\mathrm{C} 11-\mathrm{H} 11 \mathrm{~B}$ & 110.4 \\
\hline $\mathrm{C} 4-\mathrm{C} 3-\mathrm{C} 2$ & $120.91(15)$ & $\mathrm{H} 11 \mathrm{~A}-\mathrm{C} 11-\mathrm{H} 11 \mathrm{~B}$ & 108.6 \\
\hline $\mathrm{C} 4-\mathrm{C} 3-\mathrm{H} 3$ & 119.5 & $\mathrm{C} 11-\mathrm{C} 12-\mathrm{C} 8$ & $105.99(14)$ \\
\hline $\mathrm{C} 2-\mathrm{C} 3-\mathrm{H} 3$ & 119.5 & $\mathrm{C} 11-\mathrm{C} 12-\mathrm{H} 12 \mathrm{~A}$ & 110.5 \\
\hline $\mathrm{C} 5-\mathrm{C} 4-\mathrm{C} 3$ & $118.45(14)$ & $\mathrm{C} 8-\mathrm{C} 12-\mathrm{H} 12 \mathrm{~A}$ & 110.5 \\
\hline
\end{tabular}




\begin{tabular}{|c|c|c|c|}
\hline $\mathrm{C} 5-\mathrm{C} 4-\mathrm{C} 7$ & $118.30(13)$ & $\mathrm{C} 11-\mathrm{C} 12-\mathrm{H} 12 \mathrm{~B}$ & 110.5 \\
\hline $\mathrm{C} 3-\mathrm{C} 4-\mathrm{C} 7$ & $123.23(14)$ & $\mathrm{C} 8-\mathrm{C} 12-\mathrm{H} 12 \mathrm{~B}$ & 110.5 \\
\hline $\mathrm{C} 6-\mathrm{C} 5-\mathrm{C} 4$ & $121.28(15)$ & $\mathrm{H} 12 \mathrm{~A}-\mathrm{C} 12-\mathrm{H} 12 \mathrm{~B}$ & 108.7 \\
\hline $\mathrm{C} 6-\mathrm{C} 5-\mathrm{H} 5$ & 119.4 & $\mathrm{O} 1-\mathrm{C} 13-\mathrm{N} 1$ & $120.78(13)$ \\
\hline $\mathrm{C} 4-\mathrm{C} 5-\mathrm{H} 5$ & 119.4 & $\mathrm{O} 1-\mathrm{C} 13-\mathrm{N} 2$ & $122.27(13)$ \\
\hline $\mathrm{C} 1-\mathrm{C} 6-\mathrm{C} 5$ & $118.98(16)$ & $\mathrm{N} 1-\mathrm{C} 13-\mathrm{N} 2$ & $116.96(13)$ \\
\hline $\mathrm{C} 1-\mathrm{C} 6-\mathrm{H} 6$ & 120.5 & $\mathrm{C} 19-\mathrm{C} 14-\mathrm{C} 15$ & $119.46(13)$ \\
\hline $\mathrm{C} 5-\mathrm{C} 6-\mathrm{H} 6$ & 120.5 & $\mathrm{C} 19-\mathrm{C} 14-\mathrm{N} 2$ & $122.10(13)$ \\
\hline $\mathrm{N} 1-\mathrm{C} 7-\mathrm{C} 4$ & $115.17(12)$ & $\mathrm{C} 15-\mathrm{C} 14-\mathrm{N} 2$ & $118.31(14)$ \\
\hline $\mathrm{N} 1-\mathrm{C} 7-\mathrm{H} 7 \mathrm{~A}$ & 108.5 & $\mathrm{C} 16-\mathrm{C} 15-\mathrm{C} 14$ & $120.32(15)$ \\
\hline $\mathrm{C} 4-\mathrm{C} 7-\mathrm{H} 7 \mathrm{~A}$ & 108.5 & $\mathrm{C} 16-\mathrm{C} 15-\mathrm{H} 15$ & 119.8 \\
\hline $\mathrm{N} 1-\mathrm{C} 7-\mathrm{H} 7 \mathrm{~B}$ & 108.5 & $\mathrm{C} 14-\mathrm{C} 15-\mathrm{H} 15$ & 119.8 \\
\hline $\mathrm{C} 4-\mathrm{C} 7-\mathrm{H} 7 \mathrm{~B}$ & 108.5 & $\mathrm{C} 17-\mathrm{C} 16-\mathrm{C} 15$ & $120.21(15)$ \\
\hline $\mathrm{H} 7 \mathrm{~A}-\mathrm{C} 7-\mathrm{H} 7 \mathrm{~B}$ & 107.5 & $\mathrm{C} 17-\mathrm{C} 16-\mathrm{H} 16$ & 119.9 \\
\hline $\mathrm{N} 1-\mathrm{C} 8-\mathrm{C} 9$ & $114.36(13)$ & $\mathrm{C} 15-\mathrm{C} 16-\mathrm{H} 16$ & 119.9 \\
\hline $\mathrm{N} 1-\mathrm{C} 8-\mathrm{C} 12$ & $114.81(13)$ & $\mathrm{C} 16-\mathrm{C} 17-\mathrm{C} 18$ & $119.51(15)$ \\
\hline $\mathrm{C} 9-\mathrm{C} 8-\mathrm{C} 12$ & $104.49(13)$ & $\mathrm{C} 16-\mathrm{C} 17-\mathrm{H} 17$ & 120.2 \\
\hline $\mathrm{N} 1-\mathrm{C} 8-\mathrm{H} 8$ & 107.6 & $\mathrm{C} 18-\mathrm{C} 17-\mathrm{H} 17$ & 120.2 \\
\hline $\mathrm{C} 9-\mathrm{C} 8-\mathrm{H} 8$ & 107.6 & $\mathrm{C} 17-\mathrm{C} 18-\mathrm{C} 19$ & $120.64(16)$ \\
\hline $\mathrm{C} 12-\mathrm{C} 8-\mathrm{H} 8$ & 107.6 & $\mathrm{C} 17-\mathrm{C} 18-\mathrm{H} 18$ & 119.7 \\
\hline $\mathrm{C} 8-\mathrm{C} 9-\mathrm{C} 10$ & $103.43(14)$ & $\mathrm{C} 19-\mathrm{C} 18-\mathrm{H} 18$ & 119.7 \\
\hline $\mathrm{C} 8-\mathrm{C} 9-\mathrm{H} 9 \mathrm{~A}$ & 111.1 & $\mathrm{C} 14-\mathrm{C} 19-\mathrm{C} 18$ & $119.83(14)$ \\
\hline $\mathrm{C} 10-\mathrm{C} 9-\mathrm{H} 9 \mathrm{~A}$ & 111.1 & $\mathrm{C} 14-\mathrm{C} 19-\mathrm{H} 19$ & 120.1 \\
\hline $\mathrm{C} 8-\mathrm{C} 9-\mathrm{H} 9 \mathrm{~B}$ & 111.1 & $\mathrm{C} 18-\mathrm{C} 19-\mathrm{H} 19$ & 120.1 \\
\hline $\mathrm{C} 10-\mathrm{C} 9-\mathrm{H} 9 \mathrm{~B}$ & 111.1 & & \\
\hline $\mathrm{C} 6-\mathrm{C} 1-\mathrm{C} 2-\mathrm{C} 3$ & $-0.2(3)$ & $\mathrm{C} 9-\mathrm{C} 10-\mathrm{C} 11-\mathrm{C} 12$ & $24.7(2)$ \\
\hline $\mathrm{C} 11-\mathrm{C} 1-\mathrm{C} 2-\mathrm{C} 3$ & $179.54(13)$ & $\mathrm{C} 10-\mathrm{C} 11-\mathrm{C} 12-\mathrm{C} 8$ & $-1.9(2)$ \\
\hline $\mathrm{C} 1-\mathrm{C} 2-\mathrm{C} 3-\mathrm{C} 4$ & $-0.2(3)$ & $\mathrm{N} 1-\mathrm{C} 8-\mathrm{C} 12-\mathrm{C} 11$ & $-147.64(15)$ \\
\hline $\mathrm{C} 2-\mathrm{C} 3-\mathrm{C} 4-\mathrm{C} 5$ & $0.4(2)$ & $\mathrm{C} 9-\mathrm{C} 8-\mathrm{C} 12-\mathrm{C} 11$ & $-21.56(18)$ \\
\hline $\mathrm{C} 2-\mathrm{C} 3-\mathrm{C} 4-\mathrm{C} 7$ & $-177.90(15)$ & $\mathrm{C} 7-\mathrm{N} 1-\mathrm{C} 13-\mathrm{O} 1$ & $-6.1(2)$ \\
\hline $\mathrm{C} 3-\mathrm{C} 4-\mathrm{C} 5-\mathrm{C} 6$ & $-0.3(2)$ & $\mathrm{C} 8-\mathrm{N} 1-\mathrm{C} 13-\mathrm{O} 1$ & $164.53(14)$ \\
\hline $\mathrm{C} 7-\mathrm{C} 4-\mathrm{C} 5-\mathrm{C} 6$ & $178.12(14)$ & $\mathrm{C} 7-\mathrm{N} 1-\mathrm{C} 13-\mathrm{N} 2$ & $173.51(12)$ \\
\hline $\mathrm{C} 2-\mathrm{C} 1-\mathrm{C} 6-\mathrm{C} 5$ & $0.4(3)$ & $\mathrm{C} 8-\mathrm{N} 1-\mathrm{C} 13-\mathrm{N} 2$ & $-15.8(2)$ \\
\hline $\mathrm{C} 11-\mathrm{C} 1-\mathrm{C} 6-\mathrm{C} 5$ & $-179.41(12)$ & $\mathrm{C} 14-\mathrm{N} 2-\mathrm{C} 13-\mathrm{O} 1$ & $-13.1(2)$ \\
\hline $\mathrm{C} 4-\mathrm{C} 5-\mathrm{C} 6-\mathrm{C} 1$ & $-0.1(2)$ & $\mathrm{C} 14-\mathrm{N} 2-\mathrm{C} 13-\mathrm{N} 1$ & $167.32(13)$ \\
\hline $\mathrm{C} 13-\mathrm{N} 1-\mathrm{C} 7-\mathrm{C} 4$ & $-81.80(16)$ & $\mathrm{C} 13-\mathrm{N} 2-\mathrm{C} 14-\mathrm{C} 19$ & $-40.2(2)$ \\
\hline $\mathrm{C} 8-\mathrm{N} 1-\mathrm{C} 7-\mathrm{C} 4$ & $106.87(15)$ & $\mathrm{C} 13-\mathrm{N} 2-\mathrm{C} 14-\mathrm{C} 15$ & $144.02(15)$ \\
\hline $\mathrm{C} 5-\mathrm{C} 4-\mathrm{C} 7-\mathrm{N} 1$ & $169.45(13)$ & $\mathrm{C} 19-\mathrm{C} 14-\mathrm{C} 15-\mathrm{C} 16$ & $-1.1(2)$ \\
\hline $\mathrm{C} 3-\mathrm{C} 4-\mathrm{C} 7-\mathrm{N} 1$ & $-12.2(2)$ & $\mathrm{N} 2-\mathrm{C} 14-\mathrm{C} 15-\mathrm{C} 16$ & $174.79(14)$ \\
\hline $\mathrm{C} 13-\mathrm{N} 1-\mathrm{C} 8-\mathrm{C} 9$ & $131.17(15)$ & $\mathrm{C} 14-\mathrm{C} 15-\mathrm{C} 16-\mathrm{C} 17$ & $1.4(2)$ \\
\hline $\mathrm{C} 7-\mathrm{N} 1-\mathrm{C} 8-\mathrm{C} 9$ & $-58.34(18)$ & $\mathrm{C} 15-\mathrm{C} 16-\mathrm{C} 17-\mathrm{C} 18$ & $-0.2(2)$ \\
\hline $\mathrm{C} 13-\mathrm{N} 1-\mathrm{C} 8-\mathrm{C} 12$ & $-108.04(16)$ & $\mathrm{C} 16-\mathrm{C} 17-\mathrm{C} 18-\mathrm{C} 19$ & $-1.3(2)$ \\
\hline $\mathrm{C} 7-\mathrm{N} 1-\mathrm{C} 8-\mathrm{C} 12$ & $62.45(18)$ & $\mathrm{C} 15-\mathrm{C} 14-\mathrm{C} 19-\mathrm{C} 18$ & $-0.3(2)$ \\
\hline $\mathrm{N} 1-\mathrm{C} 8-\mathrm{C} 9-\mathrm{C} 10$ & $163.14(14)$ & $\mathrm{N} 2-\mathrm{C} 14-\mathrm{C} 19-\mathrm{C} 18$ & $-176.06(14)$ \\
\hline $\mathrm{C} 12-\mathrm{C} 8-\mathrm{C} 9-\mathrm{C} 10$ & $36.79(18)$ & $\mathrm{C} 17-\mathrm{C} 18-\mathrm{C} 19-\mathrm{C} 14$ & $1.5(2)$ \\
\hline $\mathrm{C} 8-\mathrm{C} 9-\mathrm{C} 10-\mathrm{C} 11$ & $-38.08(18)$ & & \\
\hline
\end{tabular}


Hydrogen-bond geometry $\left(\AA,{ }^{\circ}\right)$

\begin{tabular}{lllll}
\hline$D-\mathrm{H} \cdots A$ & $D-\mathrm{H}$ & $\mathrm{H} \cdots A$ & $D \cdots A$ & $D-\mathrm{H} \cdots A$ \\
\hline $\mathrm{N} 2-\mathrm{H} 2 N \cdots \mathrm{O} 1^{\mathrm{i}}$ & $0.828(19)$ & $2.081(19)$ & $2.8838(17)$ & $163.1(17)$ \\
\hline
\end{tabular}

Symmetry code: (i) $-x+1 / 2, y+1 / 2, z$. 